\title{
Information from CTC measurements for metastatic breast cancer prognosis-we should do more than selecting an "optimal cut point"
}

\author{
T. Fehm • W. Sauerbrei
}

Received: 2 January 2010/Accepted: 19 January 2010/Published online: 4 February 2010

(C) Springer Science+Business Media, LLC. 2010

Circulating tumor cells (CTCs) are discussed as a new prognostic marker in metastatic breast cancer. Fifty to $80 \%$ of metastatic patients have circulating tumor cells in the blood circulation [1-3]. The most currently used approach for CTC detection is the CellSearch ${ }^{\mathrm{TM}}$ system which is an semiautomated device based on immunofluorescence and flow cytometry. CTCs are isolated by immunomagnetic beads coated with antibodies against the epithelial cell adhesion molecule (EpCAM) and identified by cytokeratinpositivity, positive nuclear staining and CD 45 negativity. In contrast to the RT-PCR based approaches (e.g. AdnaTest), CTCs can be quantified.

Cristofanilli et al. [1] used the first 102 patients (training set) from a prospective multi-center trial to select a cutoff point for CTC count to stratify patients into two groups with good or poor clinical outcome and used the 75 subsequently enrolled patients as a validation set. Aiming for the clearest separation, thresholds of 1-10,000 CTCs were systematically investigated in the training set, with progression-free survival (PFS) as outcome of interest. The cut-off of 5 cells of $7.5 \mathrm{ml}$ blood resulted in a good separation between the two groups in the training and validation set for PFS and overall survival (OS). Therefore, in clinical trials (e.g. SWOG S0500) the cut-off of 5 cells is used to

This is an invited commentary to article doi: 10.1007/s10549-009-0668-7

\section{T. Fehm $(\bowtie)$}

Department of Gyn./OB, University of Tübingen, Calwer Str. 7,

72076 Tübingen, Germany

e-mail: tanja.fehm@med.uni-tuebingen.de

W. Sauerbrei

Institute of Medical Biometry and Informatics, University

Medical Center Freiburg, 79104 Freiburg, Germany discriminate patients with a poor clinical outcome from those with good clinical outcome and to modify treatment decisions.

The approach used in the paper by Cristofanilli et al. [3] to determine an appropriate cut-off is frequently used in prognostic studies. Converting continuous variables into categorical variables and selecting the cut-point that corresponds to the most significant relation with prognosis ("minimum $p$ value approach") simplifies statistical analysis. No assumption about the relationship between the continuous variable and clinical outcome has to be made prior analysis. However, dichotomization has several severe disadvantages [4] including loss of information and power, uncertainty in defining a suitable cut-point, underestimation of the extent of variation in risk, and with only two groups it is impossible to detect any non linearity in the relation between the variable and outcome. The "minimum $p$-value approach" has the additional disadvantage of overestimating the size of the effect and resulting in much too small p-values. Instead of categorizing a continuous variable, Royston et al. [4] proposed to determine a suitable functional relationship between a continuous marker and the outcome. However, considering several cut-points (e.g. 3-5) gives a step function which is often suitable to exhibit the main information in the data. The cut-off points should be selected based on biological reasons, methods of determination and simplicity.

In the current article by Botteri et al. [1] CTCs were determined in 80 metastatic breast cancer patients by the CellSearch $^{\mathrm{TM}}$ assay. Four categories of CTC values ( 0 cells, 1-4 cells, 5-20 cells and more than 20 cells) were defined and then related to progression-free and overall survival. In addition, a restricted cubic spline regression model was applied to estimate the functional relationship between CTC and survival. A non-linear increase could be 
observed in both progression and death with increasing numbers of CTCs. In a multivariable model adjusting for CA 15-3, HER2 and type of metastatic sites the risk of progression and death increased with the increasing number of CTCs but the increase lessened after 5 cells. For overall survival, the increase rate became negligible after approx. 15-20 CTCs. Similar results were obtained in univariate analyses shown as Kaplan-Meier curves for the four categories. All these analyses are a step in the right direction and underline that CTCs probably carry more than the prognostic information summarized in the two groups determined by 5 cells as the cutoff.

The difficulty of measuring the continuous marker of main interest is a further issue of CTC studies. The plateau which has been reached after five cells cannot be explained from a biological point of view. This observation may be due to the detection method of CTCs rather than to biological observations as discussed by Tibbe et al. [5]. Based on his assumptions and the statistical analysis, uncertainties in the blood collection, assay efficiency and the variability between the readers are responsible for the current threshold of five CTCs. Elimination of the errors made in the identification process of CTCs would reduces the CTC threshold from five to one CTC per $7.5 \mathrm{ml}$ blood based on his statistical considerations. Therefore, detection of a single CTC would be an identifier for worse prognosis independent on the exact number of cells.

Although the study by Botteri et al. [1] exhibits some interesting new information about the prognostic value of CTCs, the study also has weaknesses. Considering that the number of events is the effective sample size, the number of patients is small $(N=80,76$ events for PFS, 44 events for OS). Missing values have reduced the sample size furthermore. In addition, the multivariable model is not adequately reported. CTCs and the number of positive lymph nodes are strongly associated, the number of nodes has a prognostic influence on PFS and OS, but they are not included in a model estimating the prognostic effect of CTCs.

These weaknesses present three key points which can be found in many prognostic studies. First, an insufficient sample size, second, critical issues when building the multivariable model and bad reporting as the final point.
Improvements for all three issues are possible. Larger sample sizes can be achieved by further collaboration, imputation methods can be used to cope with missing values [6] and some proposals for multivariable model building have been reported and should improve the analysis of prognostic marker studies [7]. The Reporting recommendations for tumor marker prognostic studies (REMARK) designed to standardize reporting of prognostic marker studies [8] can be followed to improve the interpretability and clinical applicability of such studies.

\section{References}

1. Botteri E, Sandri MT, Bagnardi V, Munzone E, Zorzino L, Rotmensz N, Casadio C, Cassatella MC, Esposito A, Curigliano G, Salvatici M, Verri E, Adamoli L,Goldhirsch A, Nolè F (2010) Modeling the relationship between circulating tumour cells number and prognosis of metastatic breast cancer. Breast Cancer Res Treat (epub)

2. Hayes DF, Cristofanilli M, Budd GT, Ellis MJ, Stopeck A, Miller MC, Matera J, Allard WJ, Doyle GV, Terstappen LW (2006) Circulating tumor cells at each follow-up time point during therapy of metastatic breast cancer patients predict progression-free and overall survival. Clin Cancer Res 2:4218-4224

3. Cristofanilli M, Budd GT, Ellis MJ, Stopeck A, Matera J, Miller MC, Reuben JM, Doyle GV, Allard WJ, Terstappen LW, Hayes DF (2004) Circulating tumor cells, disease progression, and survival in metastatic breast cancer. N Engl J Med 351:781-791

4. Royston P, Altman DG, Sauerbrei W (2006) Dichotomizing continuous predictors in multiple regression: a bad idea. Stat Med $25: 127-141$

5. Tibbe AG, Miller MC, Terstappen LW (2007) Statistical considerations for enumeration of circulating tumor cells. Cytometry A 71:154-162

6. Sterne JAC, White IR, Carlin JB, Spratt M, Royston P, Kenward MG, Wood AM, Carpenter JR (2009) Multiple imputation for missing data in epidemiological and clinical research: potential and pitfalls. British Med J 339:157-160

7. Royston P, Sauerbrei W (2008) Multivariable model-building: a pragmatic approach to regression analysis based on fractional polynomials for modelling continuous variables. Wiley, Chichester

8. McShane LM, Altman DG, Sauerbrei W, Taube SE, Gion M, Clark GM for the Statistics Subcommittee of the NCI-EORTC Working Group on Cancer Diagnostics (2006) Reporting recommendations for tumor marker prognostic studies (REMARK). Breast Cancer Res Treat 100: 229-35 